\title{
A New Variant of the SEIZ Model to Describe the Spreading of a Rumor
}

\author{
Raul Isea ${ }^{1, ~ *, ~ K a r l ~ E . ~ L o n n g r e n ~}{ }^{2}$ \\ ${ }^{1}$ Institute of Advanced Studies- IDEA, Hoyo de la Puerta, Baruta, Venezuela \\ ${ }^{2}$ Department of Electrical and Computer Engineering, University of Iowa, Iowa City, USA
}

Email address:

raul.isea@gmail.com (R. Isea)

${ }^{*}$ Corresponding author

\section{To cite this article:}

Raul Isea, Karl E. Lonngren. A New Variant of the SEIZ Model to Describe the Spreading of a Rumor. International Journal of Data Science and Analysis. Vol. 3, No. 4, 2017, pp. 28-33. doi: 10.11648/j.ijdsa.20170304.12

Received: August 16, 2017; Accepted: September 9, 2017; Published: October 17, 2017

\begin{abstract}
We propose a variant of the SEIZ mathematical model originally proposed by Bettencourt et al, where the rumor spreads between two different scenarios $Z_{1}$ and $Z_{2}$ which do not share information with each other. This model is studied analytically where we include a new parameter $\mu$ for forgetting a rumor. We show results that were obtained using this model for a certain case in Venezuela. Finally, we also provide the numerical code for the numerical integration in order to provide a useful tool for others in their investigations.
\end{abstract}

Keywords: Rumor, SEIZ, Information, Analytical, Mathematical Model

\section{Introduction}

Information is dynamic today thanks to advances in information technology. Twitter ${ }^{\circledR}$ began just a decade ago. At the moment, Twitter ${ }^{\circledR}$ does not restrict or moderate the type of content that can be widely spread. Therefore it behaves as an information channel because it has the advantage of being able to include links to Internet pages, photos and videos which is similar to other social networks.

It is true that a social network is one that allows the exchange of data among a wide spectrum of users who have decided to establish a mutual conversation with each other. Twitter ${ }^{\circledR}$ also makes it possible to disseminate data between people who accept or not accept a request to track information. For example, Biz Stone (one of the co-founders of the platform whose Twitter account is @biz) receives information of only 719 accounts and until May 2017, had shared its messages with almost three million users.

The veracity of the information that is disseminated by Twitter ${ }^{\circledR}$ as well as other social networks, can only be validated through the news channels or "authorized sources of information" that confirm or deny the content that is propagated through the Technological platforms.

Based on the above caveat, it is necessary to develop mechanisms to understand how to quantify the spread of rumors because of the rapid advances in information technology. Eventually, it should be possible to establish criteria in order to determine a degree of veracity in the received messages.

This work begins with a review of the model proposed by Bettencourt and collaborators [1] and extends this model to the case when the rumor diffuses between two different groups of people. Finally, we show an example where applied this model with data obtained in Venezuela.

\section{Model of Bettencourt et al}

Bettencourt and collaborators [1] proposed the SEIZ model (acronyms of Susceptible, Exposed, Infected and Skeptical) to study the spread of rumors by comparing the rumor with the spreading of a disease. It starts from the idea that the potential population that is vulnerable to the rumor $S$ can become infected with this knowledge. This group can believe the news and may subsequently diffuse it $I$ until it finally reaches another group, the Skeptics $Z$, who may decide to stop further propagation.

The following aspects should be taken into account:

(i) The rate of people prone to believe the rumor is denoted by the letter $p$. 
(ii) The probabilities that characterize the exposed and skeptical populations are defined using the parameters $b$ and $l$.

(iii) The parameter $\mu$ indicate the probability of forgetting a rumor. This parameter is included for the first time in this type of model.

Thus, the system described by the SEIZ model is:

$$
\begin{gathered}
\frac{d S}{d t}=-\frac{\beta S I}{T}-b S \frac{Z}{T}-\mu S \\
\frac{d E}{d t}=\frac{(1-p) \beta S I}{T}+\frac{(1-l) b S Z}{T}-\rho E \frac{I}{T}-\mu E \\
\frac{d I}{d t}=p \beta S \frac{I}{T}+\rho E \frac{I}{T}-\mu I \\
\frac{d Z}{d t}=l b S \frac{Z}{T}-\mu Z
\end{gathered}
$$

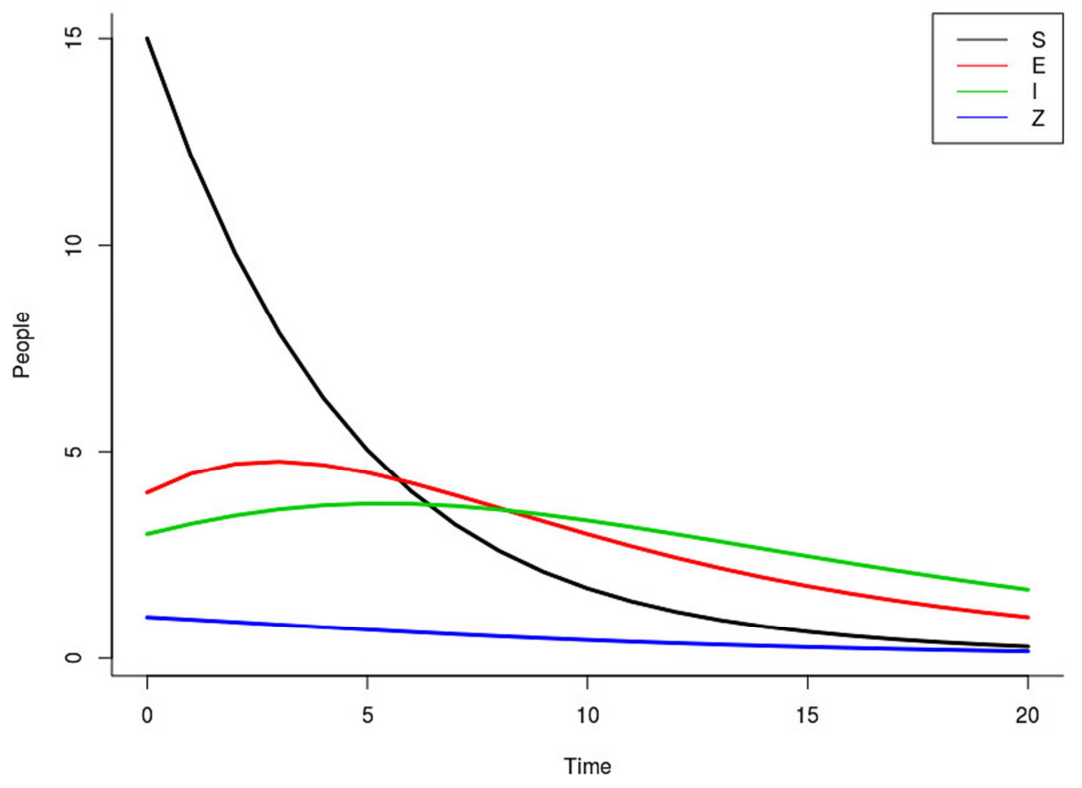

Figure 1. The numerical solution of the SEIZ model according to the values indicated in the text.

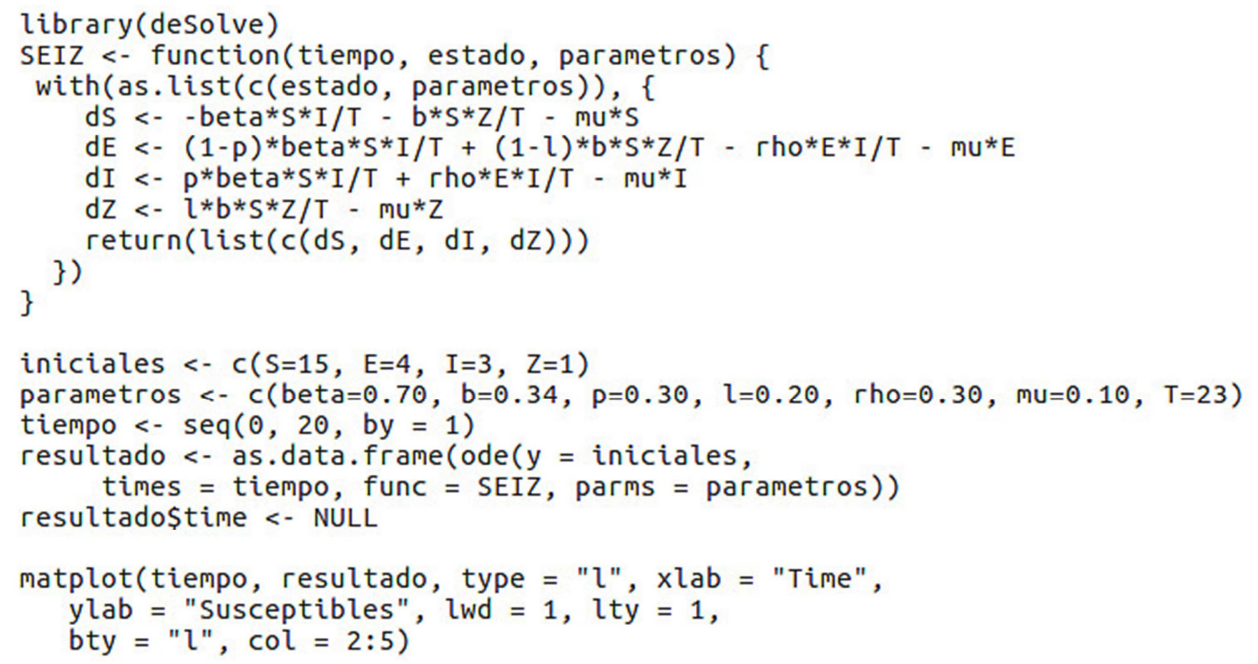

Figure 2. The numerical code in the language $R$ that resolve the differential equation (1).

The methodology to solve this set of equations has been used to explain the process of diffusion of epidemics [2, 3, 4, 5], cancer research [6] as well as in rumor theory $[7,8,9,10]$. The solution of the system of equations (1) is a bit more laborious than in the cases already published and it is possible to derive four critical points for this system with their respective eigenvalues:

Critical point $P_{1}$ :

$$
S^{*}=0, E^{*}=0, I^{*}=0, Z^{*}=0 .
$$

The only eigenvalue is $-\mu$
Critical point $P_{2}$ :

$$
S^{*}=0, E^{*}=\frac{\mu T}{\rho}, I^{*}=\frac{-\mu T}{\rho}, Z^{*}=0 .
$$

The eigenvalues are $\mu,-\mu,-\mu \frac{(\rho-\beta)}{\rho}$

Critical pointt $P_{3}$ :

$$
S^{*}=\frac{\boldsymbol{\mu}(\boldsymbol{\rho}-\boldsymbol{\beta}) \boldsymbol{T}}{\boldsymbol{\beta}(\boldsymbol{\rho}-\boldsymbol{\beta} \boldsymbol{p})}, E^{*}=\frac{-\mu T(p-1)}{\rho-\beta p}, I^{*}=\frac{-\mu T}{\beta}, Z^{*}=0 .
$$

The eigenvalues are $\mu,-\mu,-\mu\left[1+\frac{b l(p-\beta)}{\beta(\beta p-\rho)}\right]$ 
Critical point $P_{4}$ :

$$
S^{*}=\frac{\mu T}{b l}, E^{*}=\frac{\mu T(l-1)}{b l}, I^{*}=0, Z^{*}=-\frac{\mu T}{b} .
$$

The eigenvalues are $\mu,-\mu, \frac{\mu[\beta p+\rho(l-1)-b l]}{b l}$

The numerical result obtained from this mathematical model is shown in Figure 1. The actual computer program using the statistical computer program's $\mathrm{R}$ is presented in Figure 2. In this case, the following initial conditions have been randomly chosen to be: $S=15, E=4, I=3$ and $Z=1$.

The chosen values for the parameters are:

$$
\begin{gathered}
\beta=0.70, \quad b=0.34, \quad p=0.30, \quad l=0.20 \\
\rho=0.30 \mu=0.10
\end{gathered}
$$

\section{New variant of SEIZ Model}

We now extend this SEIZ model to examine the situation where the same rumor $S$ is propagated between two different groups of people that we will denote as $I_{1}$ and $I_{2}$, the goal of this manuscript. The model assumes that the same rumor is spread by two different groups of people, and we include the parameter $\mu$ for an indication of forgetting the rumor. In this case, the equations would be given by:

$$
\begin{gathered}
\frac{d S}{d t=-} \frac{\beta_{1} S I_{1}}{T}-b S \frac{Z_{1}}{T}-\frac{\beta_{2} S I_{2}}{T}-b S \frac{Z_{2}}{T}-\mu S \\
\frac{d E_{1}}{d t}=\frac{\left(1-p_{1}\right) \beta_{1} S I_{1}}{T}+\frac{\left(1-l_{1}\right) b S Z_{1}}{T} \\
-\rho E_{1} \frac{I_{1}}{T}-\mu E_{1} \\
\frac{d E_{2}}{d t}=\frac{\left(1-p_{2}\right) \beta_{2} S I_{2}}{T}+\frac{\left(1-l_{2}\right) b S Z_{2}}{T} \\
-\rho E_{2} \frac{I_{2}}{T}-\mu E_{2} \\
\frac{d I_{1}}{d t}=p_{1} \beta_{1} S \frac{I_{1}}{T}+\rho E_{1} \frac{I_{1}}{T}-\mu I_{1} \\
\frac{d I_{2}}{d t}=p_{2} \beta_{2} S \frac{I_{2}}{T}+\rho E_{2} \frac{I_{2}}{T}-\mu I_{2} \\
\frac{d Z_{1}}{d t}=l_{1} b S \frac{Z_{1}}{T}-\mu Z_{1} \\
\frac{d Z_{2}}{d t}=l_{2} b S \frac{Z_{2}}{T}-\mu Z_{2}
\end{gathered}
$$

As in the previous case, we find 8 critical points that are indicated with their respective eigenvalues:

Critical point $\mathrm{P}_{1}$ :

$$
\begin{gathered}
S^{*}=0, E_{1}^{*}=0, E_{2}^{*}=0, I_{1}^{*}=0, I_{2}^{*}=0, \\
Z_{1}^{*}=0, Z_{2}^{*}=0
\end{gathered}
$$

The only eigenvalue is $-\mu$.

Critical point $\mathrm{P}_{2}$ :

$$
\begin{gathered}
S^{*}=0, E_{1}^{*}=\frac{\mu T}{\rho}, E_{2}=0, I_{1}^{*}=-\frac{\mu T}{\rho}, I_{2}^{*}=0, \\
Z_{1}^{*}=0, Z_{2}^{*}=0
\end{gathered}
$$

The eigenvalues are $-\mu, \mu,-\frac{\mu\left(\rho-\beta_{1}\right)}{\rho}$

Critical point $\mathrm{P}_{3}$ :

$$
\begin{aligned}
& S^{*}=\frac{\mu\left(\rho-\beta_{1}\right) T}{\beta_{1}\left(\rho-p_{1} \beta_{1}\right)}, E_{1}^{*}=-\frac{\mu\left(p_{1}-1\right) T}{\rho-p_{1} \beta_{1}}, E_{2}^{*}=0, \mathrm{pc} \\
& I_{1}^{*}=-\frac{\mu T}{\beta_{1}}, I_{2}^{*}=0, Z_{1}^{*}=0, Z_{2}^{*}=0
\end{aligned}
$$

The eigenvalues are $-\mu, \mu, \frac{\mu\left(\rho-\beta_{1}\right)}{\beta_{1}}$,

$$
\begin{gathered}
\frac{\mu\left[\beta_{1}\left(\beta_{1} p_{1}-\beta_{2} p_{2}\right)+\rho\left(\beta_{2} p_{2}-\beta_{1}\right)\right]}{\beta_{1}\left(\rho-\beta_{1} p_{1}\right)}, \\
\frac{\mu\left[\beta_{1}\left(\beta_{1} p_{1}-b l_{1}\right)+\rho\left(b l_{1}-\beta_{1}\right)\right]}{\beta_{1}\left(\rho-\beta_{1} p_{1}\right)}, \\
\frac{\mu\left[\beta_{1}\left(\beta_{1} p_{1}-b l_{2}\right)+\rho\left(b l_{1}-\beta_{1}\right)\right]}{\beta_{1}\left(\rho-\beta_{1} p_{1}\right)}
\end{gathered}
$$

Critical point $P_{4}$ :

$$
\begin{gathered}
S^{*}=0, E_{1}^{*}=0, E_{2}^{*}=\frac{\mu T}{\rho}, I_{1}^{*}=0, I_{2}^{*}=-\frac{\mu T}{\rho}, \\
Z_{1}^{*}=0, Z_{2}^{*}=0
\end{gathered}
$$

The eigenvalues are $-\mu, \mu,-\frac{\mu\left(\rho-\beta_{2}\right)}{\rho}$

Critical point $P_{5}$ :

$$
\begin{gathered}
S^{*}=\frac{\mu\left(\rho-\beta_{2}\right) T}{\beta_{2}\left(\rho-p_{2} \beta_{2}\right)}, E_{1}^{*}=0, E_{2}^{*}=-\frac{\mu\left(p_{2}-1\right) T}{\rho-p_{2} \beta_{2}}, \\
I_{1}^{*}=0, I_{2}^{*}=-\frac{\mu T}{\beta_{2}}, Z_{1}^{*}=0, Z_{2}^{*}=0
\end{gathered}
$$

The eigenvalues are $-\mu, \mu, \frac{\mu\left(\rho-\beta_{2}\right)}{\beta_{2}}$,

$$
\begin{gathered}
\frac{\mu\left[\beta_{2}\left(\beta_{2} p_{2}-\beta_{1} p_{1}\right)+\rho\left(\beta_{1} p_{1}-\beta_{2}\right)\right]}{\beta_{2}\left(\rho-\beta_{2} p_{2}\right)}, \\
\frac{\mu\left[\beta_{2}\left(\beta_{2} p_{2}-b l_{2}\right)+\rho\left(b l_{2}-\beta_{2}\right)\right]}{\beta_{2}\left(\rho-\beta_{2} p_{2}\right)}, \\
\frac{\mu\left[\beta_{2}\left(\beta_{2} p_{2}-b l_{1}\right)+\rho\left(b l_{1}-\beta_{2}\right)\right]}{\beta_{2}\left(\rho-\beta_{2} p_{2}\right)}
\end{gathered}
$$

Critical point $P_{6}$ :

$$
\begin{gathered}
S^{*}=0, E_{1}^{*}=\frac{\mu T}{\rho}, E_{2}^{*}=\frac{\mu T}{\rho}, I_{1}^{*}=-\frac{\mu T}{\rho}, \\
I_{2}^{*}=-\frac{\mu T}{\rho}, Z_{1}^{*}=0, Z_{2}^{*}=0
\end{gathered}
$$

The eigenvalues are $-\mu, \mu,-\frac{\mu\left(\rho-\beta_{1}-\beta_{2}\right.}{\rho}$

Critical point $P_{7}$ :

$$
\begin{gathered}
S^{*}=\frac{\mu T}{b l_{1}}, E_{1}^{*}=\frac{\mu\left(l_{1}-1\right) T}{b l_{1}}, E_{2}^{*}=0, I_{1}^{*}=0, \\
I_{2}^{*}=0, Z_{1}^{*}=-\frac{\mu T}{b}, Z_{2}^{*}=0
\end{gathered}
$$


The eigenvalues are $-\mu, \mu, \frac{\mu\left(\beta_{1} p_{1}+\rho\left(l_{1}-1\right)-l b_{1}\right.}{b l_{1}}$,

$$
\frac{\mu\left(\beta_{2} p_{2}-b l_{1}\right)}{b l_{1}}, \frac{\mu\left(l_{2}-l_{1}\right)}{l_{1}},
$$

Critical point $P_{8}$ :

$$
\begin{gathered}
S^{*}=\frac{\mu T}{b l_{2}}, E_{1}^{*}=0, E_{2}^{*}=\frac{\mu\left(l_{2}-1\right) T}{b l_{2}}, I_{1}^{*}=0, \\
I_{2}^{*}=0, Z_{1}^{*}=0, Z_{2}^{*}=-\frac{\mu T}{b}
\end{gathered}
$$

The eigenvalues are $-\mu, \mu, \frac{\mu\left(\beta_{2} p_{2}+\rho\left(l_{2}-1\right)-l b_{2}\right.}{b l_{2}}$,

$$
\frac{\mu\left(\beta_{1} p_{1}-b l_{2}\right)}{b l_{2}}, \frac{\mu\left(l_{1}-l_{2}\right)}{l_{2}},
$$

Numerical results that have been obtained using the same statistical R program with the arbitrarily chosen initial values for the variables and the results are shown in Figure 3.
In this case, the randomly chosen initial conditions are:

$$
\begin{gathered}
S=15, E_{1}=5, E_{2}=4, I_{1}=3, \\
I_{2}=2, Z_{1}=1, Z_{2},=1 .
\end{gathered}
$$

Also chosen random values for the following parameters:

$$
\begin{gathered}
\beta_{1}=0.7, \beta_{2}=0.5=0.34, \quad b=0.3, p_{1}=0.2, \\
p_{2,}=0.1, l_{1}=0.1, l_{2},=0.3, \rho=0.1, \mu=0.1 .
\end{gathered}
$$

Here we see how the population where the rumor spreads (red) decreases, while the groups of people identified as $S, E_{1}, E_{2}, I_{1}, I_{2}, Z_{1}, Z_{2}$ are represented in green, magenta, light blue and dark blue, fuchsia and gray respectively. Figure 4 shows the code in the $\mathrm{R}$ language that allowed to solve the system of differential equations (2).

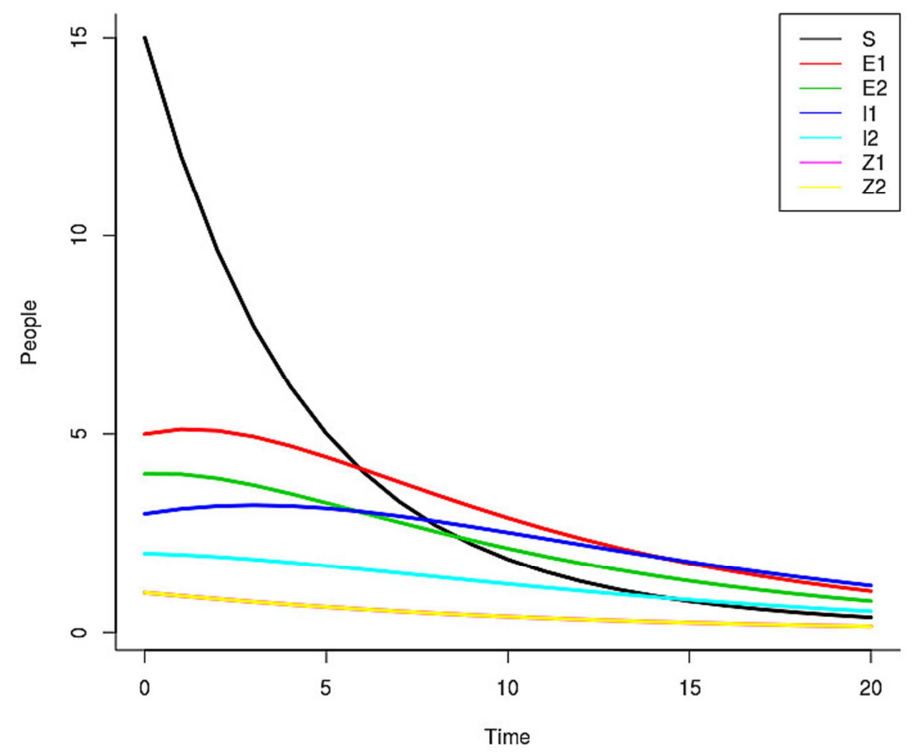

Figure 3. The numerical solution of the SEIZ model according to the values indicated in the text.

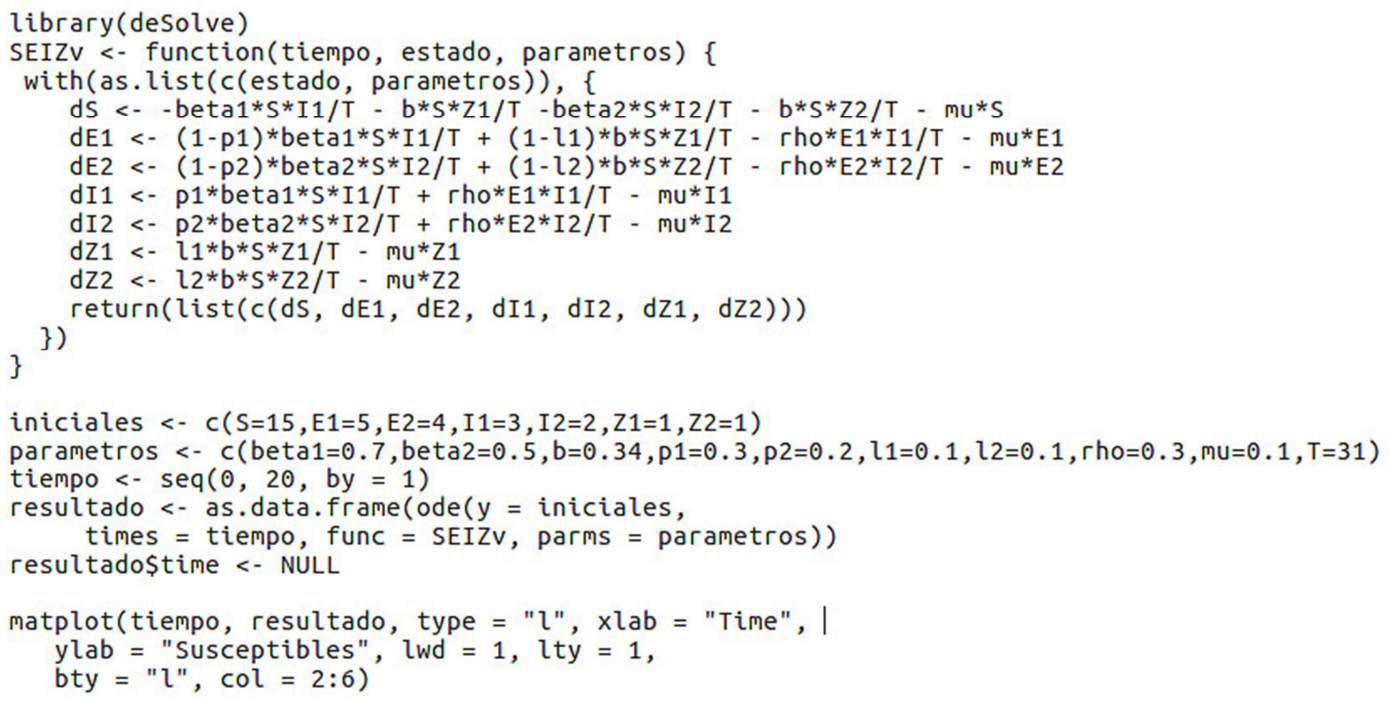

Figure 4. Code in the statistical language R for solving equations (2). 
Finally, we consider an example where we will apply this theory of the spreading of a rumor as an example (Figure 5). We show the results obtained from a search of the trends on the internet about "Sanciones en Venezuela" (translate in English: sanctions on Venezuela) averaging these values every hour (from 6 am until $12 \mathrm{~m}$, occurred at 27 august 2017).

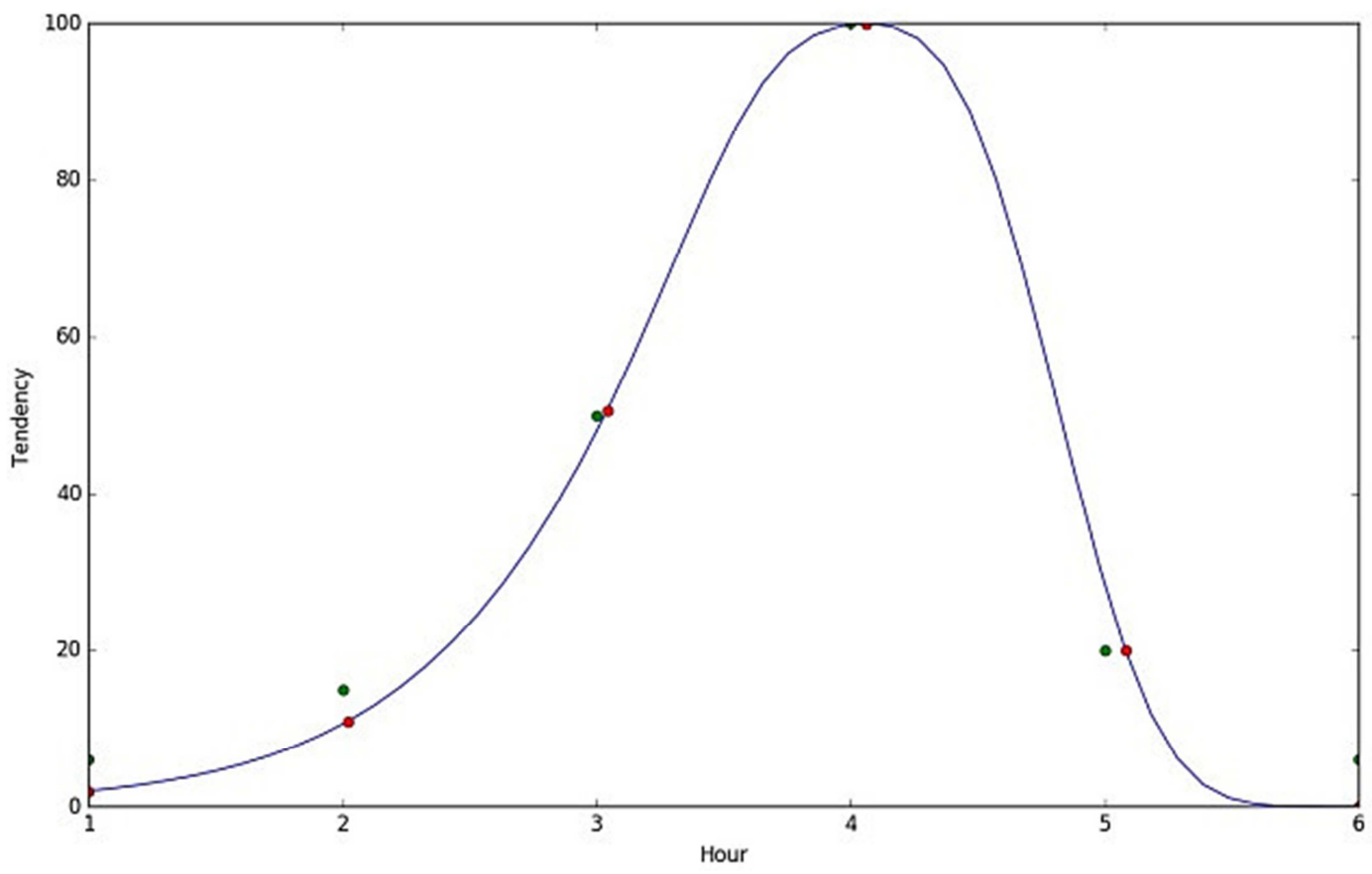

Figure 5. Least-squares fitting process of the system of equations (2) where we average the tendency on the internet about "Sanciones en Venezuela" every hour.

For this example, we must average the results hourly, because the values presented on the internet were obtained every 5 minutes and they vary significantly. We are unaware of the influence of averaging the results instead of using the data obtained on the internet.

We have considered two groups of people who are against the Sanctions on Venezuela (indicated with the subscript 1), and those people who are in favor of the sanctions (subscript 2 ). The result of the averages is shown with a green dot and that obtained from the least squares adjustment employed Python software is in red in the figure 5.

The result obtained for this period of time has been as follows: $\beta_{1}=1.79, \beta_{2}=0.07, p_{1}=1.19, p_{2}=17.67$, $l_{1}=1.84, l_{2}=1.35$. This application is an example where the possible utility of applying this type of rumor model is shown.

\section{Conclusions}

The present work poses a mathematical model based on the proposal of Bettencourt and collaborators where a rumor is propagated between two groups of different people. This model describes the spreading of a rumor or comment between two political trends, or to analysis a rumor between a possible decision between two separated communities. Although this model was considered for two groups of people, it can be generalized for any number of groups of people.

The importance of being able to present models to examine the spreading of a rumor is that a computational strategy must be designed and implemented in order to identify and evaluate any information that is being disseminated through any means of communication.

Finally, we suggest that the code in $\mathrm{R}$ can be used to integrate the system of equations so that it can help to other scientific works that wish to explore and to improve the mathematical models raised in the present work.

\section{Acknowledgements}

This paper is dedicated to the memory of Elizabeth Gámez who died on September 2017.

\section{References}

[1] L. Bettencourt, L. Cintrón-Arias, D. I. Kaiser and C. Castillo-Chávez C, "The power of a good idea: Quantitative modeling of the spread of ideas from epidemiological models", Physica A, vol. 364, pp. 513-536, 2006.

[2] R. Isea, "Analysis of an SEIR-SEI four-strain epidemic dengue model with primary and secondary Infections", Revista Electrónica Conocimiento Libre y Licenciamiento, vol. 6, pp. 3-7, 2014.

[3] R. Isea, K. E. Lonngren, “A Preliminary Mathematical Model for the Dynamic Transmission of Dengue, Chikungunya and Zika", American Journal of Modern Physics and Application, vol. 3, pp. 11-15, 2016.

[4] M. T. S. Point, H. M. Mora, A. Cortés, "The susceptible-infectious model of disease expansion analyzed and neighbor rules", Computer Science \& Information Technology, vol. 7, pp. 1-10, 2017. 
[5] J. D. Velez, R. B. Villeta, R. N. Padua, R. Hao, M. Colina, "A Predictive Model on the Spread of HIV in Cebu City", Recoletos Multidisciplinary Research Journal, vol. 3, pp. 1-8, 2017.

[6] R. Isea, K. E. Lonngren, “A Mathematical Model of Cancer under Radiotherapy", International Journal of Public Health Research, vol.3, pp. 340-344, 2015

[7] R. Isea, R. Mayo-Garcia, "Mathematical analysis of the spreading of a rumor among different subgroups of spreaders", Pure and Applied Mathematics Letters vol. 2015, pp. 50-54, 2015.
[8] R. Isea, "Análisis matemático de la difusión de un rumor entre dos grupos de personas", Revista Electrónica Conocimiento Libre y Licenciamiento, vol. 8, pp. 85-89, 2015.

[9] A. P. Mikhailov, A. P. Petrov, O. G. Proncheva, "Modeling the effect of political polarization on the outcome of propaganda battle", Computational Mathematics and Information Technologies, vol. 1, pp. 65-81, 2017.

[10] D. Aldila, H. P. Paramartha, H. Tasman, "An analysis of rumor spreading model with contra productive intervention", International Journal of Pure and Applied Mathematics, vol. 112, pp. 519-530, 2017. 